hep-th/0007110

$\mathrm{IP} / \mathrm{BBSR} / 2000-27$

\title{
Triality of Four Dimensional Strings and Networks
}

\author{
Alok Kumar円, Aalok Misra?, \\ Institute of physics, \\ Bhubaneswar 751 005, India
}

\begin{abstract}
We apply the string-triality [1] to argue the existence of the stringnetworks of solitonic $T$ and $U$-strings in the heterotic theory on $T^{6}$, $S$ and $T$-strings in IIB on $K 3 \times T^{2}$ and, $S$ and $U$-strings in IIA on $K 3 \times T^{2}$. We then show the existence of the above heterotic string networks by analyzing the supersymmetry property of the supergravity solutions. The consistency of these networks with supersymmetry in the case of IIA and IIB theories is also argued. Our results therefore give further evidence in favor of the string-triality in four dimensions.
\end{abstract}

\footnotetext{
${ }^{1}$ e-mail: kumar@iopb.res.in

${ }^{2}$ e-mail: aalok@iopb.res.in
} 
Heterotic string theory on $T^{4}$ is related by string/string duality to type IIA theory on $K 3$ [2]-[12]. This also leads to a duality among these theories, upon their compactifications to four dimensions [1]. This duality interchanges the axion-dilaton modulus of one theory with the Kähler modulus of the dual theory. Combining it with mirror symmetry (that interchanges the Kähler modulus with the complex structure modulus) 13 leads to string/string/string triality [1] relating types IIA, IIB theories on $K 3 \times T^{2}$ and heterotic string theory on $T^{4} \times T^{2}$. Let $\mathcal{T}_{X Y Z}, \mathcal{T} \equiv H, A, B$ denote any of the three theories with $S \equiv$ the axion-dilaton modulus, $T \equiv$ Kähler modulus and $U \equiv$ complex structure modulus. Then mirror symmetry takes $H_{S T U}$ to $H_{S U T}$.

It is known that the equations of motion of the heterotic strings admits a solution called an $S$-string[1, 8, 14]. Further, string-string duality takes $H_{S T U}$ to $A_{T S U}$, which under mirror symmetry goes to $B_{T U S}$. Then $A_{T S U}$ and $B_{T U S}$ admit a solution called a $T$-string [1, 8, 14] Finally, under stringstring duality, $B_{T U S}$ goes to $B_{U T S}$, which under mirror symmetry goes to $A_{U S T}$. Accordingly, equations of motion of $B_{U T S}$ and $A_{U S T}$ admit a solution called a $U$-string. More precisely, string-triality requires the following relations among various string solutions: $S / T$-strings of the heterotic theory are mapped to $T / S$-strings in IIA (on $K 3 \times T^{2}$ ), with $U$-string mapped to itself. Similarly, $S / U$-strings of the heterotic theory are mapped to $U / S$ strings in IIB on $K 3 \times T^{2}$ and $T$-string maps to itself. Finally, $T / U$-strings of IIA (on $K 3 \times T^{2}$ ) are mapped to $U / T$-strings in IIB, with $S$-string going to an $S$-string of IIB. The string tensions of $(S, T U)$-strings in the heterotic, IIA and IIB theories are expected to map into each other under the above string-dualities.

These statements carry over to the string-networks [21] - 27] as well. To find out the kind of string-networks that exist in each of these theories, we start with the IIB case. In this case, we argue that string-network of ' $(p, q)$ $S$-strings' exists in IIB on $K 3 \times T^{2}$. To construct the $(p, q)$-multiplet of such strings, we notice that the $S$-string in IIB is the dimensionally reduced ten-dimensional F-stringf. This string couples, in four dimensions, to the dilaton and axion fields of the compactified IIB theory. These fields, in turn, are the dimensional reduced version of the dilaton and NS-NS sector 2-form

\footnotetext{
${ }^{3}$ Strictly speaking, this is a 'smeared' or delocalized string solution, independent of the coordinates along $K 3 \times T^{2}$.
} 
fields in ten dimensions. Now, since the $S L(2, Z)$ S-duality of type IIB is preserved under geometric compactifications, such as $K 3 \times T^{2}$, one can form an $S L(2, Z)$ multiplet of such S-strings using their connection with the Fstring of IIB. The argument supporting the existence of such a string-network in the type IIB side then predicts the existence of the network of ' $U$-strings' in the heterotic theory. These strings couple to scalars, identified as the $U$-moduli on $T^{2}$ formed out of metric components on $T^{2}$ after removing a determinant factor. More precisely, the metric on $T^{2}$ is written as:

$$
G \equiv e^{\rho-\sigma}\left(\begin{array}{cc}
e^{-2 \rho}+c^{2} & -c \\
-c & 1
\end{array}\right)
$$

then $U \equiv c+i e^{-\rho}[1]$. Then using IIA/Heterotic duality, where $U$-moduli of one of these theories are mapped to that of the other one, we observe that ' $U$-string' networks should exist in type IIA theories as well. Furthermore, using the mapping between IIA and IIB, where $T$ and $U$ are interchanged, with $T \equiv B_{45}+i \sqrt{\operatorname{det}\left(G_{a b}\right)}$, one finds that a network of $T$-strings should exist in type IIB on $K 3 \times T^{2}$. This using IIB/heterotic duality then implies a network of $T$-strings in the heterotic case. Finally, using IIA/heterotic duality one has a network of $S$-strings in type IIA theories. To summarize, the string/string/string-triality in four dimensions implies string-networks of the $(p, q)$-multiplets of $S$ and $T$-strings in type IIB theories, $T$ and $U$-strings in the heterotic theories and $S$ and $U$-strings in type IIA theories. In this paper, we will verify that such string-networks indeed exist in these theories. We show this first for the hetetotic theory explicitly. The existence of string networks in other cases is argued on the basis of their constructions in higher dimensions.

We now start with a review of the string solutions of the heterotic theory. $S$-string solution is given by t]

$$
\begin{array}{r}
d s^{2}=e^{\eta}\left(-(d t)^{2}+\left(d x^{1}\right)^{2}\right)+\left(d r^{2}+r^{2} d \theta^{2}\right), \\
S=S_{1}+i S_{2}=a+i e^{-\eta}=\frac{1}{2 \pi i} \ln \frac{z}{r_{0}},
\end{array}
$$

\footnotetext{
${ }^{4}$ Modification of $S, T, U$-string solutions to obtain finite energy solutions, by using modular invariant functions, is given in [14]. However, since this solution reduces to the ones given below in appropriate limit, the supersymmetry condition from these solutions 14] is expected to be consistent with the ones written in this paper.
} 
where $z=x_{2}+i x_{3}\left(x_{2,3}\right.$ being the coordinates transverse to the string worldsheet coordinates $x_{0,1}$ ), ' $a$ ' is the axion field in four dimensions defined in terms of the 2-form field $B_{\mu \nu}$ as: $\epsilon^{\mu \nu \rho \lambda} \partial_{\lambda} a=\sqrt{-g} e^{-\eta} g^{\mu \nu 1} g^{\nu \nu_{1}} g^{\rho \rho_{1}} H_{\mu_{1} \nu_{1} \rho_{1}} . \eta$ is the $D=4$ dilaton. $T$-string solution is given by:

$$
\begin{gathered}
d s^{2}=-(d t)^{2}+\left(d x^{1}\right)^{2}+e^{-\sigma(r)}\left(d r^{2}+r^{2} d \theta^{2}\right) \\
T=b+i e^{-\sigma}=\frac{1}{2 \pi i} \ln \frac{z}{r_{0}},
\end{gathered}
$$

where $b$ and $\sigma$ are defined as: $T=B_{45}+i \sqrt{\operatorname{det}\left(G_{a b}\right)} \equiv b+i e^{-\sigma}$. The $U$-string solution is given by:

$$
\begin{gathered}
d s^{2}=-(d t)^{2}+\left(d x^{1}\right)^{2}+e^{-\rho(r)}\left(d r^{2}+r^{2} d \theta^{2}\right), \\
U=c+i e^{-\rho}=\frac{1}{2 \pi i} \ln \frac{z}{r_{0}} .
\end{gathered}
$$

Solutions in II A/B theories can be obtained from these by string-triality. We refer to the soultions in eqns.(2), (3), (四) as $(1,0) S$-string, $(1,0) T$-string and $(1,0) U$-string respectively. More general solutions, referred as $(p, q) S$, $T, U$-strings can be generated from these by applying appropriate $S L(2, Z)$ tranformations. We refer to them as $S L(2, Z)_{H}$. These $S L(2, Z)$ transformations in turn are the mappings of the $S L(2, Z)_{B} S$-duality transformations of the ten-dimensional type IIB theories. More precisely, the $S L(2, Z)$ of IIB in ten-dimensions can be identified as a group of constant coordinate tranformation involving any two of the six compact coordinates in the heterotic theory. All these $S L(2, Z)$ 's are in fact part of the $O(6,22, Z) T$-duality symmetry of the heterotic string, belonging to its diagonal subgroup $S L(6, Z)$. The mapping from $S L(2, Z)_{B}$ to $S L(2, Z)_{H}$ is obtained by identifying their actions on various fields in IIB and heterotic theories. in four dimensions. Field content of the heterotic theory has a metric $\left(g_{\mu \nu}\right)$, scalars: $S$-modulus defined earlier, 28 vector fields transforming in a vector representation of $O(6,22)$ and 132 additional scalars forming a coset: $O(6,22) / O(6) \times O(22)$. To identify the appropriate $S L(2, Z)$, we denote indices $(0,1,2,3)$ as $(\mu, \nu)$. Internal indices are denoted as: $a \equiv(4,5), i \equiv(6, . .9), m=(7,8,9)$. In the IIB case below, we will denote the internal $K 3$ by indices $(\bar{I}, \bar{J})$ etc..

In the IIB theory, the fields corresponding to the heterotic theory mentioned in the last paragraph are obtained from the $K 3 \times T^{2}$ compactification of the ten dimensional fields. The metric $\left(g_{\mu \nu}\right)$ comes from the components 
of the ten-dimensional metric. 2 gauge fields $\left(A_{\mu}\right.$ 's) are the Kaluza-Klein modes on $T^{2}: g_{\mu a}, B_{\mu a}^{1}, B_{\mu a}^{2}$. Remaining 22 gauge fields $\left(A_{\mu}\right.$ 's) arise from the self-dual 4-form in ten dimensions: 3 come from the self-dual and 19 from the anti-self-dual 2-forms on $K 3$. Among scalars, 2 are the ten-dimensional axion-dilaton fields, 3 come from the components of metric along $T^{2}$, and 58 scalars are the geometric moduli of $K 3$. The 2 -form fields give scalars from the components along $T^{2}:\left(B_{a b}^{1}, B_{a b}^{2}\right)$ as well as from the space-tiem components $\left(B_{\mu \nu}^{1}, B_{\mu \nu}^{2}\right)$. They also give rise to 22 scalars each from $B_{\bar{I} \bar{J}}^{1}, B_{\bar{I} \bar{J}}^{2}$. The 4 -form field gives another 23 scalars, out of which 22 come from the 2 -forms on $K 3$ and one from 0-form. Combining these one gets an identical field content as in the heterotic theory.

To obtain a precise connection between $S L(2, Z)_{B}$ and $S L(2, Z)_{H}$, we now discuss the tranformation properties of above fields, both in IIB and heterotic theories. In the IIB case, 4 gauge fields arising as $\left(B_{\mu a}^{1}, B_{\mu a}^{2}\right)$ tranform as a vector under $S L(2, Z)_{B}$. The remaining ones, coming from the ten-dimensional metric: $\left(g_{\mu a}\right)$ and the self-dual 4-form field, are neutral under this $S L(2, Z)$. In the heterotic theory, since the $S L(2, Z)$ is realized as a coordinate transformation on any two of the compact coordinates mentioned above (for definiteness we choose them as $x^{5}$ and $x^{6}$ ), four gauge fields transforming as a vector under this $S L(2, Z)$ are identified as:

$$
\left(\begin{array}{c}
g_{\mu 5} \\
g_{\mu 6}
\end{array}\right),\left(\begin{array}{c}
B_{\mu 5} \\
B_{\mu 6}
\end{array}\right)
$$

It is evident that the remaining gauge fields: $A_{\mu}^{I}, g_{\mu 4}, B_{\mu 4}, g_{\mu m}, B_{\mu m}$ are neutral under this $S L(2, Z)$ in the heterotic theory as well. As a result the action of $S L(2, Z)_{B}$ and $S L(2, Z)_{H}$ are identical on vector fields in two theories.

We now compare the actions of the two $\operatorname{SL}(2, Z)$ 's on scalars in these theories. In the type IIB case, the axion-dilaton combination forms a coset $S L(2) / S O(2)$. In the heterotic theory, this role is played by the complex structure associated with directions $\left(x^{5}, x^{6}\right)$. In addition, in the IIB theory in $D=4$, one also has scalar fields transforming in vector representation of $S L(2, Z)_{B}$. These scalars are:

$$
\left(\begin{array}{c}
B_{a b}^{1} \\
B_{a b}^{2}
\end{array}\right),\left(\begin{array}{c}
B_{\bar{I} \bar{J}}^{1} \\
B_{\bar{I} \bar{J}}^{2}
\end{array}\right), \quad\left(\begin{array}{c}
B_{\mu \nu}^{1} \\
B_{\mu \nu}^{2}
\end{array}\right) .
$$

\footnotetext{
${ }^{5}$ leaving the combination $\left(x^{4}, x^{5}\right)$, which is already used in defining the $T^{2}$ mentioned above.
} 
The remaining scalars: $g_{a b}, g_{I J}$, as well as the ones coming from the self-dual 4-form, are invariant under $S L(2, Z)$. In the heterotic theory, the role of the scalars transforming as a vector (analogous to the ones in (6)) is played by field components:

$$
\left(\begin{array}{c}
g_{m 5} \\
g_{m 6}
\end{array}\right), \quad\left(\begin{array}{c}
B_{m 5} \\
B_{m 6}
\end{array}\right), \quad\left(\begin{array}{c}
A_{5}^{I} \\
A_{6}^{I}
\end{array}\right), \quad\left(\begin{array}{c}
g_{45} \\
g_{46}
\end{array}\right), \quad\left(\begin{array}{c}
B_{45} \\
B_{46}
\end{array}\right),
$$

and the invariant scalars are: $\operatorname{det}(G), B_{45}, g_{m n}, B_{m n}, g_{m 4}, B_{m 4}, A_{m}^{I}, A_{4}^{I}, \phi, B_{\mu \nu}$, $g_{44}$, where $G$ is the metric in the $\left(x^{5}, x^{6}\right)$-space. Their numbers match exactly with the ones in IIB theory.

We have therefore obtained the mapping of the $S L(2, Z) S$-duality transformation of IIB theory to the heterotic one. The $S L(2, Z)_{H}$ symmetry of the heterotic theory can now, in principle, be used for generating of the $(p, q)$ type solution for $S, T$ or $U$-strings starting from the $(1,0)$ solutions that we wrote earlier in eqns.(4)-(3). As classical solutions, these are equivalent, related by coordinate transformations. However, each one of them play a different role in a network construction that we discuss below, due to the differences in their Killing spinor conditions. Our aim will be to write down the conditions satisfied by the Killing spinors for the classical $(p, q)$-string backgrounds generated in this manner. For this we will follow the strategy in [15, 21, 22] to write down the supersymmetry of the $(1,0)$ solution first, and then obtain the supersymmetry property of the $(p, q)$-solution by applying $S L(2, Z)_{H}$ transformation. We will find some crucial differences among the three cases, with important implications for their network constructions.

We now start by explicitly evaluating the killing spinor equations in the $S, T, U$-string backgrounds (2)-(3). The supersymmetric transformations of the gravitino $\psi_{\mu}$, dilatino $\lambda$ and gaugino $\chi$, (in the absence of background gauge fields) for $\mathrm{D}=4$ heterotic string theory is given by [16, 1]

$$
\begin{aligned}
& \delta \psi_{\mu}=\left[\nabla_{\mu}-\frac{1}{8} H_{\mu \nu \rho} \Gamma^{\nu \rho}+\frac{1}{4} Q_{\mu}^{\hat{m} \hat{n}} \Gamma^{\hat{m} \hat{n}}\right] \epsilon, \\
& \delta \lambda=\frac{1}{4 \sqrt{2}}\left[\gamma^{\mu} \frac{\partial_{\mu}\left(S_{2}-i \gamma^{5} S_{1}\right)}{S_{2}}\right] \epsilon=0, \\
& \delta \chi^{\hat{m}}=\frac{1}{\sqrt{2}} \gamma^{\mu} P_{\mu}^{\hat{m} \hat{n}} \Gamma^{\hat{n}} \epsilon,
\end{aligned}
$$

where $\gamma^{\hat{5}} \equiv \frac{1}{4 !} \epsilon_{\hat{\mu} \hat{\nu} \hat{\rho} \hat{\lambda}} \gamma^{\hat{\mu}} \gamma^{\hat{\nu}} \gamma^{\hat{\rho}} \gamma^{\hat{\lambda}}, Q_{\mu}^{\hat{m} \hat{n}} \equiv\left(V_{R} L \partial_{\mu} V_{R}^{T}\right)^{\hat{m} \hat{n}}$ and $P_{\mu}^{\hat{m} \hat{n}} \equiv\left(V_{L} L \partial_{\mu} V_{R}^{T}\right)^{\hat{m} \hat{n}}$. $V_{L}$ and $V_{R}$ are defined using $\mathrm{O}(6,22)$ matrix of moduli $M: M=V^{T} V=$ 
$V_{R}^{T} V_{R}+V_{L}^{T} V_{L}=\frac{1}{2}(M+L)+\frac{1}{2}(M-L)$ where $L$ is an $\mathrm{O}(6,22)$-invariant metric. Then

$$
V=\left(\begin{array}{c}
V_{R} \\
V_{L}
\end{array}\right)=\frac{1}{\sqrt{2}}\left(\begin{array}{cc}
E^{-1} & E^{T} \\
E^{-1} & -E^{T}
\end{array}\right) .
$$

Hatted/unhatted Greek letters denote four-dimensional tangent space/curved space indices, and Hatted/unhatted Latin letters denote six-dimensional tangent space/curved space indices. The covariant derivative in (8) is given by:

$$
\nabla_{\mu}=\partial_{\mu}+\frac{1}{4} \omega_{\mu}^{\hat{\mu} \hat{\nu}} \Gamma_{\hat{\mu} \hat{\nu}}
$$

with $\omega_{\mu}^{\hat{\mu} \hat{\nu}}$ being the spin connection. We now obtain the supersymmetry conditins for ' $(1,0)$ ' $S, T$ and $U$-strings.

\section{(I) $(1,0) S$-string}

The (1.0) $S$-string solution is given in equation (2). We now evaluate the supersymmetric variations for this background. One can now rewrite

$$
-\frac{1}{8} H_{\mu \nu \rho} \Gamma^{\nu \rho}=-\frac{1}{8 S_{2}^{2} \sqrt{-g}} \gamma_{\hat{5}}^{\hat{r} \hat{\theta}} \Gamma_{\mu \nu} \partial^{\nu} a
$$

where $\gamma_{\hat{\zeta}}^{\hat{r} \hat{\theta}}=\gamma_{\hat{0}} \gamma_{\hat{1}} \gamma_{\hat{r}} \gamma_{\hat{\theta}}$ The spin connection $\omega_{\mu}^{\hat{\mu} \hat{\nu}}$ for the S-string is given by:

$$
\omega_{\mu}^{\hat{\mu} \hat{\nu}}=\left(\begin{array}{cccc}
0 & 0 & \frac{\delta_{\mu}^{0}\left(\partial_{r} \eta(r)\right) e^{\eta(r) / 2}}{2} & 0 \\
0 & 0 & \frac{\delta_{\mu}^{1}\left(\partial_{r} \eta(r)\right) e^{\eta(r) / 2}}{2} & 0 \\
-\frac{\delta_{\mu}^{0}\left(\partial_{r} \eta(r)\right) e^{\eta(r) / 2}}{2} & -\frac{\delta_{\mu}^{1}\left(\partial_{r} \eta(r)\right) e^{\eta(r) / 2}}{2} & 0 & -\frac{\delta_{\mu}^{\theta}}{r} \\
0 & 0 & \frac{\delta_{\mu}^{\theta}}{r} & 0
\end{array}\right),
$$

where entries in the above matrix are w.r.t. indices $(\hat{0}, \hat{1}, \hat{r}, \hat{\theta})$. The killing spinor equations obtained from non-trivial variations of the gravitino are then given by

$$
\begin{aligned}
& (i) \delta \psi_{0}=\partial_{0} \epsilon-\frac{1}{4} e^{3 \eta(r) / 2}\left(\partial_{r} \eta(r)\right) e^{-\eta(r)} \gamma^{\hat{r}} \gamma^{\hat{0}}\left(-1-\gamma^{\hat{0}} \gamma^{\hat{1}}\right) \epsilon=0 \\
& (i i) \delta \psi_{1} \sim \delta \psi_{0}
\end{aligned}
$$




$$
\begin{aligned}
& (i i i) \delta \psi_{r}=\partial_{r} \epsilon-\frac{1}{4} \partial_{r} \eta(r) \gamma^{\hat{0}} \gamma^{\hat{1}} \epsilon=0 \\
& (i v) \delta \psi_{\theta}=\partial_{\theta} \epsilon-\frac{1}{2 r} \gamma_{\hat{r}} \gamma_{\hat{\theta}} \epsilon=0
\end{aligned}
$$

Thus, using $\frac{\gamma_{\hat{r}} \gamma_{\hat{\theta}}}{r}=\gamma^{\hat{2}} \gamma^{\hat{3}}$ (which has eigenvalues \pm 1 ), and $e^{-\eta(r)}=-\frac{1}{2 \pi} \ln \frac{r}{r_{0}}$, $a(\theta)=\frac{\theta}{2 \pi}$, one sees that (23) can be consistently solved for $\epsilon$ to give:

$$
\epsilon(r, \theta)=e^{-\frac{\eta(r)}{4}} \cdot e^{\frac{1}{2} \gamma^{\hat{2}} \gamma^{\hat{3}} \theta} \epsilon_{0},
$$

and satisfies

$$
\left(1+\gamma^{\hat{0}} \gamma^{\hat{1}}\right) \epsilon_{0}=0
$$

Solutions like the one in (14) have been discussed eariler in [29]). The supersymmetric variation of the dilatino is given by:

$$
\begin{aligned}
& \delta \lambda=\frac{e^{\eta(r)}}{4 \sqrt{2}}\left[\gamma^{\hat{r}} \partial_{r}\left(e^{-\eta(r)} \epsilon+i \gamma^{\hat{5}} \gamma^{\hat{\theta}} \partial_{\theta} a(\theta)\right)\right] \epsilon \\
& =\frac{e^{\eta(r)}}{4 \sqrt{2}}\left(1+\gamma^{\hat{0}} \gamma^{\hat{1}}\right) \epsilon=0,
\end{aligned}
$$

which, as consistency would require, gives the same chirality condition as (15). The supersymmetric variation of the gaugino is trivially zero.

(II) $\underline{(1,0) \text { U string }}$

The (1.0) U-string solution is given in equation (4). The supersymmetric variation of the dilatino for (4) is trivially zero. The variations of the gravitino and gaugino are given by:

$$
\begin{aligned}
& \delta \psi_{\mu}=\left[\nabla_{\mu}+\frac{1}{4} Q_{\mu}^{\hat{m} \hat{n}} \Gamma^{\hat{m} \hat{n}}\right] \epsilon=0, \\
& \delta \chi^{\hat{m}}=\frac{1}{\sqrt{2}} \gamma^{\mu} P_{\mu}^{\hat{m} \hat{n}} \Gamma^{\hat{m}} \epsilon=0 .
\end{aligned}
$$

where $Q_{\mu}$ and $P_{\mu}$ were defined earlier. The spin connection $\omega_{\mu}^{\hat{\mu} \hat{\nu}}$ for (雨) is given by:

$$
\omega_{\mu}^{\hat{\mu} \hat{\nu}}=\left(\begin{array}{cccc}
0 & 0 & 0 & 0 \\
0 & 0 & 0 & 0 \\
0 & 0 & 0 & -\frac{\delta_{\mu}^{\theta}}{r}\left(1-\frac{r}{2} \partial_{r} \rho(r)\right) \\
0 & 0 & \frac{\delta_{\mu}^{\theta}}{r}\left(1-\frac{r}{2} \partial_{r} \rho(r)\right) & 0
\end{array}\right) .
$$


Now, to evaluate $Q_{\mu}$ and $P_{\mu}$ in (17), it is sufficient for our purpose to consider only the $T^{2}$ that is common to $T^{4} \times T^{2}$ and $K 3 \times T^{2}$. Hence, for (4), the matrix $M$ is given by:

$$
M=\left(\begin{array}{cc}
G^{-1} & 0 \\
0 & G
\end{array}\right),
$$

where $G=E E^{T}$ is the metric on $T^{2}$ as given in (11), for unit determinant (i.e. $\sigma=0$ in (11)) with

$$
E=\left(\begin{array}{cc}
-e^{-\frac{\rho}{2}} & c e^{\frac{\rho}{2}} \\
0 & -e^{\frac{\rho}{2}}
\end{array}\right)
$$

Thus, one gets:

$$
\begin{aligned}
& Q_{\mu}^{\hat{m} \hat{n}}=\left(V_{R} L \partial_{\mu} V_{R}^{T}\right)^{\hat{m} \hat{n}}=\frac{1}{2}\left(E^{-1} \partial_{\mu} E+E^{T} \partial_{\mu} E^{T-1}\right)^{\hat{m} \hat{n}} \\
& =\left(\begin{array}{cc}
0 & -\frac{e^{\rho}}{2}\left(\partial_{\mu} c\right) \delta_{\mu}^{\theta} \\
\frac{e^{\rho}}{2}\left(\partial_{\mu} c\right) \delta_{\mu}^{\theta} & 0
\end{array}\right),
\end{aligned}
$$

with matrix components denote indices $\hat{m}, \hat{n}$. Similarly,

$$
\begin{aligned}
& \gamma^{\mu} P_{\mu}^{\hat{m} \hat{n}}=\left(\gamma^{\mu} V_{L} L \partial_{\mu} V_{R}^{T}\right)^{\hat{m} \hat{n}}=\frac{1}{2} \gamma^{\mu}\left(E^{-1} \partial_{\mu} E-E^{T} \partial_{\mu} E^{T-1}\right)^{\hat{m} \hat{n}} \\
& =\frac{1}{2}\left(\begin{array}{cc}
-\gamma^{\hat{r}} \partial_{r} \rho(r) & -e^{\rho(r)} \gamma^{\hat{\theta}} \partial_{\theta} c(\theta) \\
-e^{\rho(r)} \gamma^{\hat{\theta}} \partial_{\theta} c(\theta) & \gamma^{\hat{r}} \partial_{r} \rho(r)
\end{array}\right) .
\end{aligned}
$$

The only non-trivial variation of the gravitino is given for $\mu=\theta$ :

$$
\begin{aligned}
& \delta \psi_{\theta}=\left(\partial_{\theta}+\frac{1}{2} \omega_{\theta}^{\hat{r} \hat{\theta}} \Gamma_{\hat{r}} \Gamma_{\hat{\theta}}+\frac{1}{2} Q_{\theta}^{\hat{4} \hat{5}} \Gamma^{\hat{4}} \Gamma^{\hat{5}}\right) \epsilon \\
& =\left(\partial_{\theta}-\frac{\gamma_{\hat{r}} \gamma_{\hat{\theta}}}{2 r}\right) \epsilon+\frac{1}{4} \partial_{r} \rho(r) \gamma_{\hat{r}} \gamma_{\hat{\theta}} \epsilon-\frac{1}{4}\left(\partial_{\theta} c(\theta)\right) e^{\rho(r)} \Gamma^{\hat{4}} \Gamma^{\hat{5}} \epsilon=0 .
\end{aligned}
$$

Using $\frac{\gamma_{\hat{r}} \gamma_{\hat{\theta}}}{r}=\gamma^{\hat{2}} \gamma^{\hat{3}}$ (which has eigenvalues \pm 1 ) and $e^{-\rho(r)}=-\frac{1}{2 \pi} \ln \frac{r}{r_{0}}, c(\theta)=$ $\frac{\theta}{2 \pi}$, one sees that (23) can be consistently solved for $\epsilon$ by imposing:

$$
\partial_{\theta} \epsilon-\frac{1}{2} \gamma^{\hat{2}} \gamma^{\hat{3}} \epsilon=0
$$

and

$$
\frac{1}{4} \partial_{r} \rho(r) \gamma_{\hat{r}} \gamma_{\hat{\theta}} \epsilon-\frac{1}{4}\left(\partial_{\theta} c(\theta)\right) e^{\rho(r)} \Gamma^{\hat{4}} \Gamma^{\hat{5}} \epsilon=0 .
$$


Equation (24) has a solution:

$$
\epsilon(\theta)=e^{\frac{\gamma^{\hat{\gamma}} \hat{\beta}}{2} \theta} \epsilon_{0}
$$

and (25) gives:

$$
\left(1+\gamma^{\hat{2}} \gamma^{\hat{3}} \Gamma^{\hat{4}} \Gamma^{\hat{5}}\right) \epsilon_{0}=0 .
$$

One can show that one gets identical condition as (27) from the variation of the gaugino. Equation (27) again implies that $1 / 2$ of spacetime supersymmetry is preserved.

\section{(III) $\underline{(1,0) \mathrm{T} \text { string }}$}

Let us consider the T string solution of (3). For this case, the matrix $M$ has the general $\mathrm{O}(2,2)$ form:

$$
M=\left(\begin{array}{cc}
G^{-1} & -G^{-1} B \\
B G^{-1} & G-B G^{-1} B
\end{array}\right),
$$

which implies:

$$
V=\left(\begin{array}{c}
V_{R} \\
V_{L}
\end{array}\right)=\frac{1}{\sqrt{2}}\left(\begin{array}{cc}
E^{-1} & E^{T}-E^{-1} B \\
E^{-1} & -E^{T}-E^{-1} B
\end{array}\right) .
$$

The $T^{2}$ metric is given by: $G=e^{-\sigma(r)} \mathbf{1}_{2}=E E^{T}$, which implies $E=e^{-\frac{\sigma(r)}{2}} \mathbf{1}_{2}$. $Q_{\mu}$ and $P_{\mu}$ are then given by:

$$
\begin{aligned}
& Q_{\mu}^{\hat{m} \hat{n}}=\frac{1}{2}\left(E^{-1} \partial_{\mu} E+E^{T} \partial_{\mu} E^{T-1}+E^{-1}\left(\partial_{\mu} B\right) E^{T-1}\right)^{\hat{m} \hat{n}} \\
& =\frac{1}{2} e^{\sigma(r)}\left(\begin{array}{cc}
0 & \partial_{\mu} b \\
-\partial_{\mu} b & 0
\end{array}\right) \delta_{\mu}^{\theta},
\end{aligned}
$$

and

$$
\begin{aligned}
& \gamma^{\mu} P_{\mu}^{\hat{m} \hat{n}}=\frac{1}{2} \gamma^{\mu}\left(E^{-1} \partial_{\mu} E+E^{T} \partial_{\mu} E^{T-1}-E^{-1}\left(\partial_{\mu} B\right) E^{T-1}\right)^{\hat{m} \hat{n}} \\
& =\frac{1}{2}\left(\begin{array}{cc}
-\gamma^{\hat{r}} \partial_{r} \sigma(r) & e^{\sigma(r)} \gamma^{\hat{\theta}} \partial_{\theta} b \\
-e^{\sigma(r)} \gamma^{\hat{\theta}} \partial_{\theta} b & -\gamma^{\hat{r}} \partial_{r} \sigma(r)
\end{array}\right) \delta_{\mu}^{\theta} .
\end{aligned}
$$


The analysis parallels that of the U string with $\rho \rightarrow \sigma$ and $c \rightarrow-b$. Thus, the supersymmetry condition is

$$
\left(1-\gamma^{\hat{2}} \gamma^{\hat{3}} \Gamma^{\hat{4}} \Gamma^{\hat{5}}\right) \epsilon_{0}=0
$$

Again, this condition is the same as the one obtained by the supersymmetric variation of the gaugino.

We have therefore obtained the supersymmetry properties of the string solution [1] of the heterotic string theory, referred to here as $(1,0)$-solutions. The $(p, q)$ - solutions can then be generated by applying the $S L(2, Z)_{H}$ discussed earlier. In the present case, as mentioned earlier, the role of this $S L(2, Z)$ is played by constant coordinate transformations acting on coordinates $x^{5}$ and $x^{6}$. These transformations generate other classical solutions, which in fact are equivalent to the original ones as they are generated by constant coordinate transformations. Also, as in the case of ten-dimensional type IIB theory, the string tension is once again given by an $S L(2, Z)$ invariant function, from which one can read tensions of strings with different quantum numbers. This expression involves moduli as well as $(p, q)$ quantum numbers denoting charges. As we will see shortly, the supersymmetry of the transformed solutions are in general different than the original ones. As a result, when one combines many such string to construct a network configuration, one has a situation where individual strings contribute differently to the total energy and supersymmetry is broken to $1 / 4$.

Now, it is clear that $S$-string mentioned earlier is invariant under the above $S L(2, Z)$. As a result, one does not have any new supersymmetry condition arising out of their action on such solutions. In fact, we already notice a crucial difference among supersymmetry conditions of $S-, T$ - and $U$ - strings given in equations (15), (27) and (32), namely that, in addition to the space-time gamma matrices that appear in equations for $S$-string, we also have internal gamma matrices appearing in the $T$ - and $U$ - string supersymmetry condition. They finally allow one to make an alignment using space-time and internal orientations of strings in order to construct string networks.

Now, to obtain the killing spinor for " $(\mathrm{p}, \mathrm{q})$ " $U$ or $T$ string, one performs a constant coordinate $\mathrm{SL}(2)_{H}$ transformation on the killing spinors in equations (27) and (32). The effect of $\mathrm{SL}(2, \mathrm{Z})$ S-duality transformations in heterotic string and $\mathrm{D}=10 \mathrm{IIB}$ theories have been studied [15, 21]. It is known 
that spinors transform as a representation of the maximal compact subgroup which in this case truns out to be parameterized by a rotation angle. In this case, this corresponds to a rotation induced by constant coordinate transformation in $\left(x^{5}, x^{6}\right)$-space. Then the new supersymmetry condition for the "(p,q)" $U$ or $T$ string will be:

$$
\left[1 \pm \gamma^{\hat{2}} \gamma^{\hat{3}} \Gamma^{\hat{4}}\left(\cos \theta \Gamma^{\hat{5}}+\sin \theta \Gamma^{\hat{6}}\right)\right] \epsilon=0
$$

( \pm for $U / T$ “(p,q)” strings) where using [28, 15],

$$
e^{i \theta}=\frac{p-q \tau^{*}}{\left|p-q \tau^{*}\right|}
$$

where $\tau$ is the complex structure generated from metric components $G_{55,56,66}$.

We now discuss the construction of string networks for the above string solutions. First, as already stated, S-string remains unchanged under $\operatorname{SL}(2, \mathrm{Z})_{H}$. Moreover, since supersymmetry condition (15) is also invariant under SL $(2, \mathrm{Z})_{H}$, one does not have any possibility to put several different types of strings together to form a network. These can however be formed using multiplets of $T$ and $U$ strings. To see this from the point of view of supersymmetry, we rewrite (33) by defing spinors which are eigenvectors of $i \Gamma^{\hat{4}} \Gamma^{\hat{5}}$, denoted by $\epsilon_{ \pm}$. Choosing the following representation for the six-dimensional $\Gamma^{\hat{m}}$ 's:

$$
\Gamma^{\hat{4}, \hat{5}, \hat{6}}=\sigma^{2,1,3} \otimes \mathbf{1}_{2} \otimes \mathbf{1}_{2},
$$

$\epsilon$ can be taken to be:

$$
\epsilon=\left(\begin{array}{c}
\tilde{\epsilon}_{+} \\
\tilde{\epsilon}_{-}
\end{array}\right)=\left(\begin{array}{c}
\epsilon_{+} \otimes \epsilon_{0} \\
\epsilon_{-} \otimes \epsilon_{0}
\end{array}\right)
$$

where $\tilde{\epsilon}_{ \pm}, \epsilon_{ \pm}$and $\epsilon_{0}$ are 16-, 8-, and 2-component spinors respectively, where.

$$
i \Gamma^{\hat{4}} \Gamma^{\hat{5}} \epsilon_{ \pm}= \pm \epsilon_{ \pm}
$$

Hence, one gets the following result from (33):

$$
\epsilon_{+}+i \epsilon_{-}= \pm i e^{-i \theta} \gamma^{\hat{2}} \gamma^{\hat{3}}\left(\epsilon_{+}-i \epsilon_{-}\right) .
$$

Similar condition can be written for $T$-string as well. The $1 / 4$ spacetime supersymmetry of the $U$ and $T$ string networks can now be established by following the arguments of [21, 22]. 
To summarize, we have now shown that by superimposing $T$ and $U$ string networks of the heterotic theory, one can have network configuration in this theory preserving only $1 / 4$ of the supersymmetry. We have therefore verified a prediction of string-triality from the point of view of string network construction in heterotic theory.

We now discuss the existence of $S$ and $T$ strings in type IIB on $K 3 \times T^{2}$, as prediced by the string-triality. First, to see the existence of $S$-string networks, we notice that the four dimensional $S$-strings are charged under the four dimensional axion-dilaton fields. Among these, the axion field orginates from the NS-NS 2-form $B_{M N}$ in ten dimensions and the dilaton comes from the ten-dimensional dilaton. As a result, the $S$ string solution mentioned earlier for IIB case, can in fact be identified with the $(1,0)$-string of the tendimensional theory. Moreover due to these identifications, the $S$-duality in four dimensions is seen to follow from the one in $D=10$. As a result, the existence of the $S$-string networks in IIB theory also follows from the $(p, q)$ string networks in ten-dimensions. Similar arguments hold for the $S$-string networks in type IIA as well. However one now has to use the fact that they are equivalent to IIB theories, when compactified on a circle.

To complete the arguments in favor of the string-triality, we now discuss the existence of $T$-string networks in IIB theories and $U$-string networks in IIA theories. By using the mapping between the two theories, when compactified on a circle, we automatically have the existence of the string network in IIA, given the one for IIB. So, finally to show the presence of $T$-string networks in IIB theories, we notice that, by promoting the solution to six dimensions, and then by using Hodge duality of 3-form field strengths, one obtains a 'smeared' or delocalized string solution in six dimensions. Promoting this further to a smeared solution in ten dimensions, one again finds the string networks in these theories. In other words, to put $S$-string solutions to an appropriate form in $D=10$ we needed to use the Hodge-duality in four dimensions, whereas in the present case we make use of it in six dimensions. In both these cases we ignore the $K 3$ factor, by writing down the smeared solutions with no coordinate dependence along $K 3$ directions.

To conclude, we have given evidence in favor of string-triality in four dimensions by explicitly examining the supersymmety properties in the heterotic theory. We have also given arguments in favor of the existence of string-networks in type II theories, that are predicted by the above triality. The results are summarized in Fig 1. 


\section{References}

[1] M.J. Duff, James T. Liu, J. Rahmfeld, Nucl. Phys. B459 (1996) 125, hep-th/9508094.

[2] M. J. Duff and J. X. Lu, Loop expansions and string/five-brane duality, Nucl. Phys. B 357 (1991) 534, hep-th/9305142.

[3] M. J. Duff and R. R. Khuri, Four-dimensional string/string duality, Nucl. Phys. B411 (1994) 473, hep-th/9305142.

[4] M. J. Duff and J. X. Lu, Black and super p-branes in diverse dimensions, Nucl. Phys. B416 (1994) 301, hep-th/9306052.

[5] M. J. Duff and R. Minasian, Putting string/string duality to the test, Nucl. Phys. B436 (1995) 507, hep-th/9406198.

[6] M. J. Duff, Classical/quantum duality, in Proceedings of the International High Energy Physics Conference, Glasgow (July 1994), (Eds. Bussey and Knowles).

[7] M. J. Duff, R. R. Khuri and J. X. Lu, String solitons, Phys. Rept259 (1995) 213, hep-th/9412184.

[8] M. J. Duff, Strong/weak coupling duality from the dual string, Nucl. Phys. B442 (1995) 47 hep-th/9501030.

[9] C. M. Hull and P. K. Townsend, Unity of superstring dualities, Nucl. Phys. B 438 (1995) 109, hep-th/9410167.

[10] E. Witten, String theory dynamics in various dimensions, Nucl. Phys. B443 (1995) 85, [hep-th/9503124].

[11] A. Sen, String string duality conjecture in six dimensions and charged solitonic strings, Nucl. Phys. B450 (1995) 103, [hep-th/9504027.

[12] J. A. Harvey and A. Strominger, The heterotic string is a soliton, Nucl. Phys. B449 (1995) 535, hep-th/9504047.

[13] M. Dine, P. Huet and N. Seiberg, Large and small radius in string theory, Nucl. Phys. B 322 (1989) 301. 
[14] A. Kehagias, N=2 Heterotic Stringy Cosmic Strings, TUM-HEP-262/96, hep-th/9611110.

[15] T. Ortin, Sl(2,R)-duality covariance of killing spinors in axion-dilaton black holes, Phys. Rev. D51 (1995) 790, hep-th/9404035.

[16] D. Youm, Black Holes and Solitons in String Theory, Phys. Rept 316 (1999) 1, hep-th/9710046.

[17] J. Schwarz, Lectures on Superstring and $M$ Theory Dualities, hepth/9607201].

[18] O. Aharony, J. Sonnenschein and S. Yankielowicz, Interactions of strings and $D$-branes from $M$ theoryInteractions of strings and $D$-branes from $M$ theory, Nucl. Phys. B474 (1996) 309 [hep-th/9603009]; O. Aharony and A. Hanany, Branes, Superpotentials and Superconformal Fixed Points, Nucl. Phys. B504 (1997) 239, hep-th/9704170; O. Aharony, A. Hanany and B. Kol, Webs of (p,q) 5-branes, Five Dimensional Field Theories and Grid Diagrams JHEP 9801(1998) 002, hep-th/9710116].

[19] K. Dasgupta and S. Mukhi, BPS Nature of 3-String Junctions Phys. Lett. B423 (1998) 261, [hep-th/9711094].

[20] S-J. Rey and J-T. Yee, BPS Dynamics of Triple $(p, q)$ String Junction, Nucl. Phys. B526 (1998) 229, hep-th/9711202.

[21] A. Sen, String Network, JHEP 9803:005 (1998), [hep-th/9711130].

[22] S. Bhattacharyya, A. Kumar and S. Mukhopadhyay, String Network and U-Duality, Phys. Rev. Lett. 81 (1998) 754, [hep-th/9801141].

[23] M. Krogh and S. Lee, String Network from M-theory, Nucl. Phys. B516 (1998) 241, [hep-th/9712050; Y. Matsuo and K. Okuyama, BPS Condition of String Junction from M theory, Phys. Lett. B426 (1998) 294, hep-th/9712070.

[24] C. Callan and L. Thorlacious, Worldsheet Dynamics of String Junctions, Nucl. Phys. B534 (1998) 121, hhep-th/9803097.

[25] S. Mukherji, On the SL(2,Z) Covariant World-Sheet Action with Sources, Mod. Phys. Lett. A 13 (1998) 2819, hep-th/9805031. 
[26] A. Kumar and S. Mukhopadhyay, U-duality and Network Configurations of Branes, Int. J. Mod. Phys.A14 (1999) 3252, hep-th/9806126.

[27] M. Gabardiel and B. Zwiebach, Exceptional groups from open strings, Nucl. Phys. B518 (1998) 151, [hep-th/9709013]; M. Gabardiel, T. Hauer and B. Zwiebach, Open string - string junction transitionsOpen string - string junction transitions, Nucl. Phys. B525 (1998) 117, hep-th/9801205; O. Bergman, Three-Pronged Strings and 1/4 BPS States in N=4 Super-Yang-Mills Theory, Nucl. Phys. B525 (1998) 104, hep-th/9712211; O. Bergman and A. Fayyazuddin, String Junctions and BPS States in Seiberg-Witten Theory Nucl. Phys. B531 (1998) 108, hep-th/9802033; O. Bergman and B. Kol, String Webs and 1/4 BPS Monopoles Nucl. Phys. B536 (1998) 149, hep-th/9804160; K. Hashimoto, H. Hata and N. Sasakura, 3-String Junction and BPS Saturated Solutions in SU(3) Supersymmetric Yang-Mills Theory Phys. Lett. B431 (1998) 303, [hep-th/9803127]; Multi-Pronged Strings and BPS Saturated Solutions in SU(N) Supersymmetric Yang-Mills Theory Nucl. Phys. B535 (1998) 83, hetp-th/9804164; P. Ramadevi, Supergravity Solution for Three-String Junction in M-Theory, JHEP 0006 (2000) 005, hep-th/9906247; A. Kumar, Charged Macroscopic type II Strings and their Networks, JHEP 9912 (1999) 001; A. Kumar, Non-Planar String Networks on Tori, JHEP 0003 (2000) 010, [hep-th/0002150]; A. Kumar and S. Mukherji, On Charged Strings and their Networks hepth/0005093]; (For a more comprehensive list of references, see) B. Kol, On the Spatial Structure of Monopoles, hep-th/0002118.

[28] J. Schwarz, An SL(2,Z) Multiplet of Type IIB Superstrings, Phys.Lett. B360 (1995) 13-18; Erratum-ibid. B364 (1995) 252 [hep-th/9508143.

[29] H. Lu, C.N. Pope, J. Rahmfeld, A Construction of Killing Spinors on $S^{n}$, J.Math.Phys. 40 (1999) 4518-4526, hep-th/9805151]; P. M. Llatas, J. M. Sanchez de Santos, S-Duality, SL(2,Z) Multiplets and Killing Spinors, Phys.Lett. B484 (2000) 306-314, hep-th/9912159. 


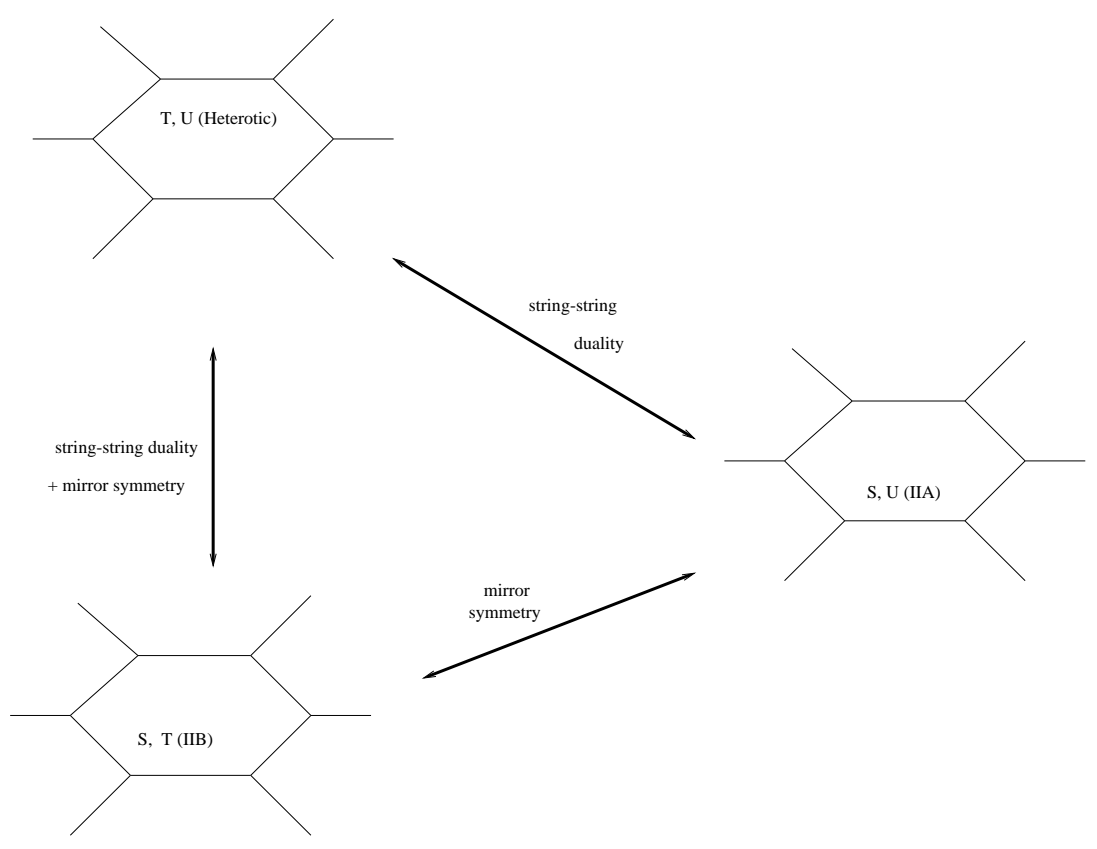

Figure 1: Triality of string networks in four dimensions 